\title{
Mediation model of comorbid anxiety disorders in late-life depression
}

\author{
Chawisa Suradom ${ }^{1}$, Nahathai Wongpakaran ${ }^{1 *}$ (D), Tinakon Wongpakaran ${ }^{1}$, Peerasak Lerttrakarnnon², \\ Surin Jiraniramai ${ }^{2}$, Unchulee Taemeeyapradit ${ }^{3}$, Surang Lertkachatarn ${ }^{4}$, Suwanna Arunpongpaisal ${ }^{5}$ \\ and Pimolpun Kuntawong ${ }^{1}$
}

\begin{abstract}
Background: A number of studies have been conducted on risk factors of comorbid anxiety disorders regarding late-life depression (LLD). This study investigated the associated factors and their relationship to comorbid anxiety disorders in LLD.

Methods: Participants included 190 elderly Thais (73.2\% female, with a mean age of $68.39 \pm 6.74$ years) with depressive disorders, diagnosed according to DSM-IV Diagnosis Axis I disorders assessed by Mini-International Neuropsychiatric Interview. Demographic data, medical and psychiatric history, family psychiatric history, past depression, family history of depression, Neuroticism Inventory and 7-Item Hamilton Depression Rating Scale (HAMD-7) were analyzed for path analysis using Structural Equation Model framework. The bootstrapping method was used for testing indirect effects.

Results: Being female was associated with comorbid anxiety disorders with an indirect effect $(\beta=-0.032, P=0.018)$ through neuroticism, depression severity, history and family history of depression. Family history of depression had no effect on comorbidity $(P=0.090)$. Neuroticism had an indirect effect on comorbid anxiety disorders $(\beta=0.075$, $P=0.019)$ via depression severity as reflected by HAMD-7 score $(\beta=0.412, P=<0.001)$. Total variance explained from this model was $11 \%$. This model had good-fit index with Chi-square $>0.05$, CFI and TLI $>0.95$ and RMSEA $<0.06$.

Conclusion: Neuroticism mediates the effect of relationship between sex, family history and history of depressive disorders and comorbid anxiety disorders in LLD. Moreover, depression severity is a mediator for neuroticism and comorbid anxiety disorders. Longitudinal studies are warranted to indicate the importance of effective treatment of depression to lower the risk of developing comorbid anxiety disorders among depressed elderly.
\end{abstract}

Keywords: Anxiety disorder, Late-life depression, Neuroticism, Mediation

\section{Background}

Anxiety is common in late-life depression (LLD), both as a symptom and as a comorbid disorder [1]. Comorbid anxiety disorders have been found to increase the burden of depression as reflected by quality of life, physical disability, increased health care use and mortality [2].

*Correspondence: nahathai.wongpakaran@cmu.ac.th

${ }^{1}$ Geriatric Psychiatry Unit, Department of Psychiatry, Faculty of Medicine, Chiang Mai University, 110 Intawaroros Rd., Chiang Mai 50200, Thailand

Full list of author information is available at the end of the article
According to related studies, comorbid anxiety disorder was associated with poorer social function, a higher level of somatic symptoms, a higher level of suicidality [1], a higher risk for alcohol abuse [3], disability [4] and lower cognitive performance [5].

A number of studies have been conducted on risk factors of comorbid anxiety disorders regarding LLD. Evidence has proved a significant correlation of history of depressive disorders and comorbid anxiety and depression. Specifically, past depressive disorders increased the odds of repeated anxiety disorders, depressive disorders 
and of repeated comorbid anxiety and depressive disorders [6].

Apart from history of depressive disorder, the associated factors of the comorbidity identified by related studies included, being female [7], higher level of depression severity [8] and personality trait neuroticism [9], while results for family histories of mental disorder as a risk were inconclusive [10], despite the fact that several studies have identified genetic correlation to the emergence of depressive and anxiety disorders [11-13]. None however, has investigated the role of family history in the comorbidity of anxiety and depressive disorders.

Notably, personality trait neuroticism is a predisposing variable to developing many psychiatric disorders, especially anxiety and depressive disorders. One particular study attempted to impose a mediation model on the theory with findings that included neuroticism substantially for a number of anxiety disorders, namely, GAD, panic disorder and phobias [9]. The role of neuroticism on depression was more clearly demonstrated in elderly populations $[14,15]$.

Based on these aforementioned associated variables, evidence showed that comorbid anxiety disorders were influenced by sociodemographic factors such as sex, biologically predisposing factors such as history of depression and family history of depression and psychological factors such as personality trait neuroticism. What is lacking is to include all these variables addressing bio-psychosocial issues and test them simultaneously.

We hypothesized that these independent variables (individual's history of depression, family history of depression and being female) would have a positively direct effect on comorbid anxiety disorders, regarded as dependent variables. We hypothesized that neuroticism and level of depression severity should serve as mediators of the relationship between independent and dependent variables; thereby, three independent variables, two mediators and a single outcome rendered eight mediational models (Fig. 1). However, as recommended by Hayes, testing all variables as a single integrated model is more worthwhile than testing each separately because it would be interesting to find the strength of association of each paired variable [16]. From the single model, we hypothesized that the level of neuroticism and depression severity would mediate the direct effect of past depression, family history of depression or sex on comorbid anxiety disorders.

Moreover, none of the related studies on the associated factors of comorbid anxiety disorders has been conducted regarding LLD. Therefore, this study aimed to

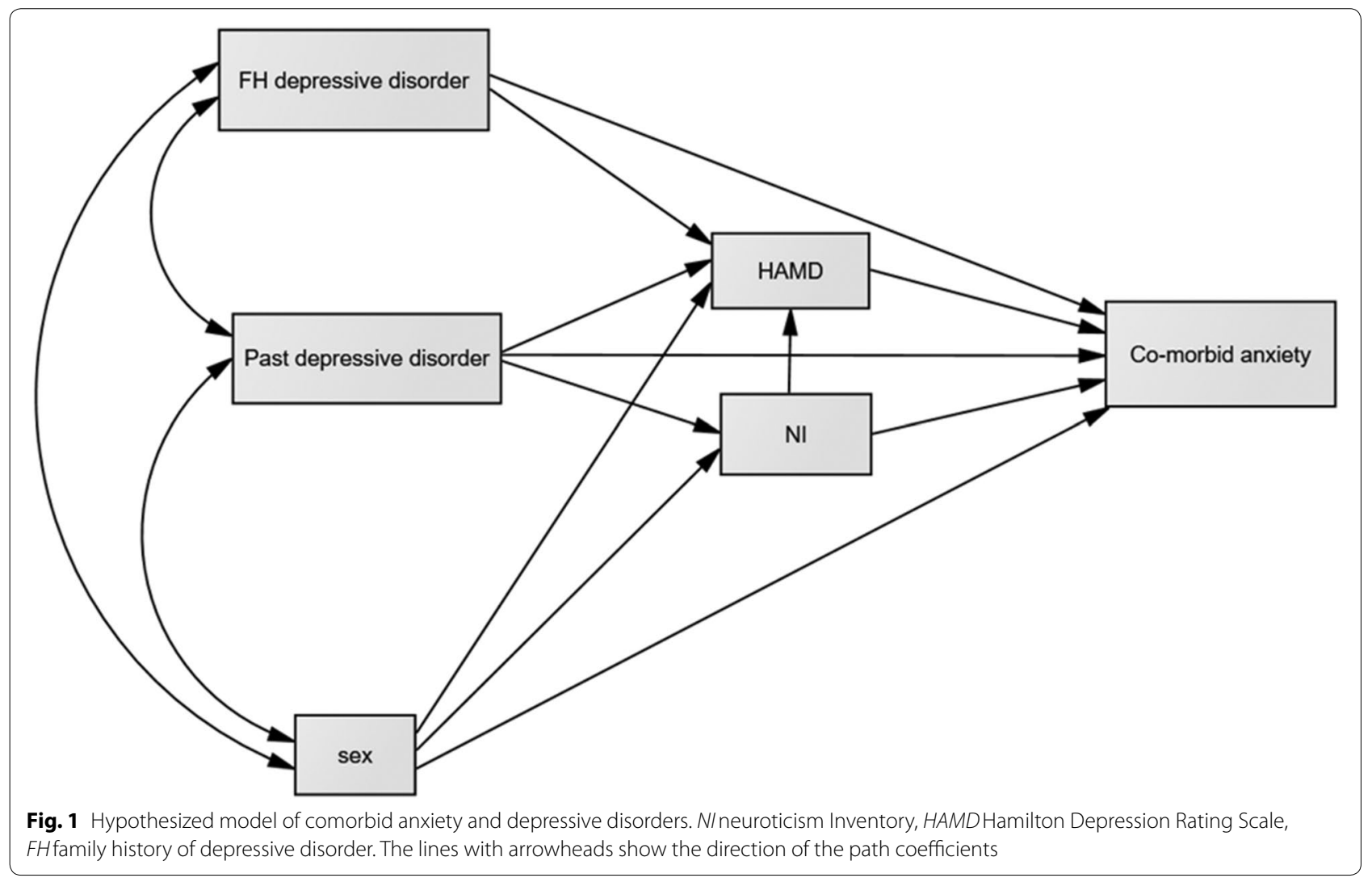


investigate the associated factors and their relationship on comorbid anxiety disorders concerning LLD.

\section{Methods}

This research comprised a secondary analysis of data of elderly Thai patients with a diagnosis of depressive disorders between October 2012 and March 2015 [17].

\section{Participants}

Psychiatric elderly patients were evaluated at four different tertiary care centers across Thailand, namely, Maharaj Nakorn Chiang Mai Hospital, Prince of Songkla University Hospital, Songkla Rajanagarindra Psychiatric Hospital and Prasat Neurological Institute using the Mini-International Neuropsychiatric Interview (MINI) to evaluate major Axis I diagnoses according to DSM-IV or ICD-10 criteria [17]. A total of 190 patients with depressive disorders were then evaluated for comorbid anxiety disorders and their associated factors.

Information regarding demographic characteristics and symptoms were gathered and evaluated to identify risk factors for comorbid anxiety disorders in LLD. Inclusion criteria included new cases of patients aged 60 and beyond who attended the psychiatric outpatient unit with one of the following symptoms: sadness, loss of interest, sleep disturbance, poor appetite, memory problems, lack of energy and unexplained medical symptoms. The exclusion criteria included those with medical conditions that interfered with the interview process, illiteracy or language barrier, cognitive impairment, history of schizophrenia, schizo-affective or mania with residual symptoms [17].

LLD is defined as depressive disorders among patients aged 60 and above, receiving treatment in tertiary care centers, as diagnosed by the Mini-International Neuropsychiatric Interview (MINI) for major depressive disorders (MDD) based on DSM-IV TR. Anxiety disorders consist of panic disorders, agoraphobia, social phobias and generalized anxiety disorders. Other related disorders included obsessive-compulsive disorders and posttraumatic stress disorders.

\section{Measurements}

Demographic data, medical and psychiatric history, family psychiatric history and a number of measurements included those listed below.

\section{Mini-International Neuropsychiatric Interview (MINI)}

Mini-International Neuropsychiatric Interview (M.I.N.I., 5.0.0) is a semi-structured interview for diagnosing psychiatric disorders based on the DSM-IV. Depressive disorders, i.e., major depressive disorders (MDD) and dysthymia, were in Modules A and B. The Thai version has been validated and widely used [18].

The 7-item Hamilton Depression Rating Scale (HAMD-7) [19]. The HAMD-7 is a semi-structured interview for diagnosing severity of depression based on the DSM-IV. We employed the GRID version of HAMD-7, for which the researchers were trained until the inter-rater agreement was ensured to reach a $100 \%$ agreement on the scoring.

\section{Neuroticism Inventory (NI) [20]}

The NI is a self-reporting assessment tool for measuring dimensionality of the neuroticism personality trait according to Eysenck's Five-factor Model [21]. The NI, developed by Wongpakaran et al., includes 15 items with a 0 to 4 Likert scale [20]. A higher score reflects a higher level of neuroticism. It has been demonstrated as a valid and reliable tool. The present study yielded a Cronbach's alpha of 0.83 .

\section{Statistical analysis}

Descriptive statistics were used to assess demographic data such as sex and age. Correlations among variables were analyzed using Pearson's, point-biserial, and Spearman's rank correlations. All demographic data, medical and psychiatric history, and measurement scores were analyzed to identify meaningful correlations. The main outcome in this study was comorbid anxiety-depressive disorder. Sociodemographic data served independent variables. Mediator variables included neuroticism and severity of depressive symptom, and significant variables were included in the model. Path analysis in the structural equation model framework was used to build the interrelationships among all variables.

Following steps were carried out. The first step involved exploring the relationship between independent and dependent variables using Pearson's and pointbiserial correlation to ensure significant relationship among variables to be candidate predictors and mediators for the comorbid anxiety-depressive disorder. Then we examined the relationship among all potential predictors using path analysis. The hypothetical relationships were evaluated to test how neuroticism and severity of depression may mediate the pathway from sociodemographic variables to comorbid anxiety-depressive disorder. Model fit was evaluated using the following criteria: Chi-square $/ d f<3$, Comparative Fit Index $>0.95$, Tucker-Lewis Index $>0.95$ and root mean square error of approximation (RMSEA) $<0.06$. In model comparison, Consistent Akaike information criterion (CAIC) was used to compare two or more not-nested models, with smaller values representing a better fit, and Bayesian Information Criteria (BIC) was 
also used to determine the best-fit model as it has a greater tendency to pick parsimonious models. Finally, we evaluated whether the putative mediators, according to the best-fit model, could significantly mediate the effect using the bias-corrected and accelerated (BCa) bootstrapped method to calculate the empirical significance level (based on 10,000 bootstrap replications).

The data were analyzed using IBM SPSS for Windows, Version 22.0 (IBM Company, Armonk, NY, USA); AMOS, Version 22 was used for the path model.

Table 1 Demographic data and current psychiatric history

\begin{tabular}{ll}
\hline Demographic data & $\mathbf{N}(\%)$ \\
\hline Female gender & $139(73.2)$ \\
Age (years), mean (SD); min-max & $68.39(6.7) ; 60-88$ \\
Marital living status & \\
Living alone & $75(39.5)$ \\
Living with spouses & $115(60.5)$ \\
Years of education, mean (SD); min-max & $6.67(5.0) ; 0-30$ \\
History of depressive disorders & $27(14.2)$ \\
Family history of depressive disorders & $7(3.7)$ \\
Concurrent medical conditions & $52(27.4)$ \\
Anxiety disorders & $32(16.8)$ \\
Panic disorder & $9(4.7)$ \\
Agoraphobia & $10(5.3)$ \\
Social phobia & $2(1.1)$ \\
OCD & $4(2.1)$ \\
PTSD & $7(3.7)$ \\
GAD & $14(7.4)$ \\
Measurements, mean (SD) & \\
MoCA & $15.70(5.473)$ \\
HAMD-7 & $10.30(6.340)$ \\
\hline NI & $53.44(11.699)$ \\
\hline
\end{tabular}

OCD obsessive compulsive disorder, PTSD post-traumatic stress disorder, GAD generalized anxiety disorder, MoCA Montreal Cognitive Assessment, NIneuroticism inventory, HAMD-77-Item Hamilton Depression Rating Scale

\section{Results}

Demographic data and history of depression are shown in Table 1. The overall prevalence of anxiety disorders, also diagnosed with MINI, in this elderly population with LLD was $16.84 \%$, with GAD and agoraphobia being the most prevalent. Significant correlated variables from literature review, as identified by Pearson's correlation, were sex, history and family history of depressive disorder, Neuroticism Inventory score, measuring neuroticism personality trait and HAMD-7 scores, measuring depression severity.

According to Baron, correlation between independent $(X)$ and outcome $(Y)$, and $X$ and $M$ has to be observed [22]. Table 2 shows significant correlations for being female and depressive history, neuroticism, comorbid anxiety disorders, and depression severity. Depressive disorder also correlated with neuroticism, comorbid anxiety disorders and depression severity. Interestingly, comorbid anxiety disorders significantly correlated with family history of depressive disorder. In contrast, neuroticism and depression severity correlated with all variables, except for family history of depressive disorder.

We hypothesized that depression severity, family and history of depressive disorder, being female and neuroticism all had an effect on comorbid anxiety disorder in LLD, while the latter four also exhibited an effect through depression severity as a mediator. Moreover, we suspected the mediation effect of neuroticism on sex and history of depressive disorder.

\section{Mediation analysis}

The proposed mediation model satisfied a good-fit model to the data with the $\chi^{2}(d f)=0.288$, RMSEA $=0.000$, $\mathrm{TLI}=1.122, \mathrm{CFI}=1.000, \mathrm{CAIC}=119.270, \mathrm{BIC}=100.270$. However, to find the best model, CAIC was used to compare other models in which nonsignificant paths were deleted one by one. The final model yielded the best fit with the lowest CAIC while showing other fit indices above the cut-off criteria $\left(\chi^{2}(d f)=1.136, \mathrm{RMSEA}=0.027\right.$,

Table 2 Zero-order correlations between variables

\begin{tabular}{lllll}
\hline Variable & Gender & $\begin{array}{l}\text { Past } \\
\text { depressive } \\
\text { disorders }\end{array}$ & $\begin{array}{l}\text { Family history } \\
\text { of depressive } \\
\text { disorders }\end{array}$ & Neuroticism \\
\hline Gender & 1 & & & Comorbid anxiety \\
Past depressive disorders & -0.173 & 1 & 1 & 1 \\
Family history of depressive disorders & -0.055 & 0.100 & 0.049 & $0.189^{* *}$ \\
Neuroticism & $-0.214^{* *}$ & $0.159^{*}$ & $0.211^{* *}$ & 1 \\
Comorbid anxiety & $-0.146^{*}$ & $0.143^{*}$ & 0.088 & $0.234^{* *}$ \\
HAMD-7 & $-0.181^{*}$ & $0.313^{* *}$ & 1 & 1
\end{tabular}

${ }^{*} p<.05,{ }^{* *} p<.01,{ }^{* * *} p<.001$; GDS Geriatric Depression Scale, HAMD-7the 7-Item Hamilton Depression Rating Scale, MoCA Montreal Cognitive Assessment 
$\mathrm{TLI}=0.977, \mathrm{CFI}=0.989, \mathrm{CAIC}=95.411, \mathrm{BIC}=81.411)$

(Fig. 2).

\section{Testing for mediation model}

From Table 3, only HAMD showed a direct effect on comorbidity of anxiety disorders in LLD $(\beta=0.215, \mathrm{BCa}$ $95 \% \mathrm{CI}=0.107-0.316)$. Neuroticism Inventory score, history of depression, and being female predicted the comorbidity of anxiety disorders in LLD with indirect effects of $\beta=-0.053, P=0.018, \beta=0.092$ (BCa 95\% $\mathrm{CI}=0.041-0.153$ ), $\beta=-0.020$ (BCa 95\% CI $=-0.046$ to -0.007$)$, respectively. Family history of depressive disorder itself had a nearly significant effect on the comorbidity $(P=0.061)$.

\section{Discussion}

The present study confirmed our hypothesis of various variables associated with the comorbidity of anxiety disorder in LLD. Firstly, depression severity plays a role on the comorbidity of anxiety disorder. Several studies have focused on the effect of anxiety on depression severity with established knowledge in terms of neuroendocrinology, especially cortisol increasing the risk of depression. No study to date, however, has mentioned

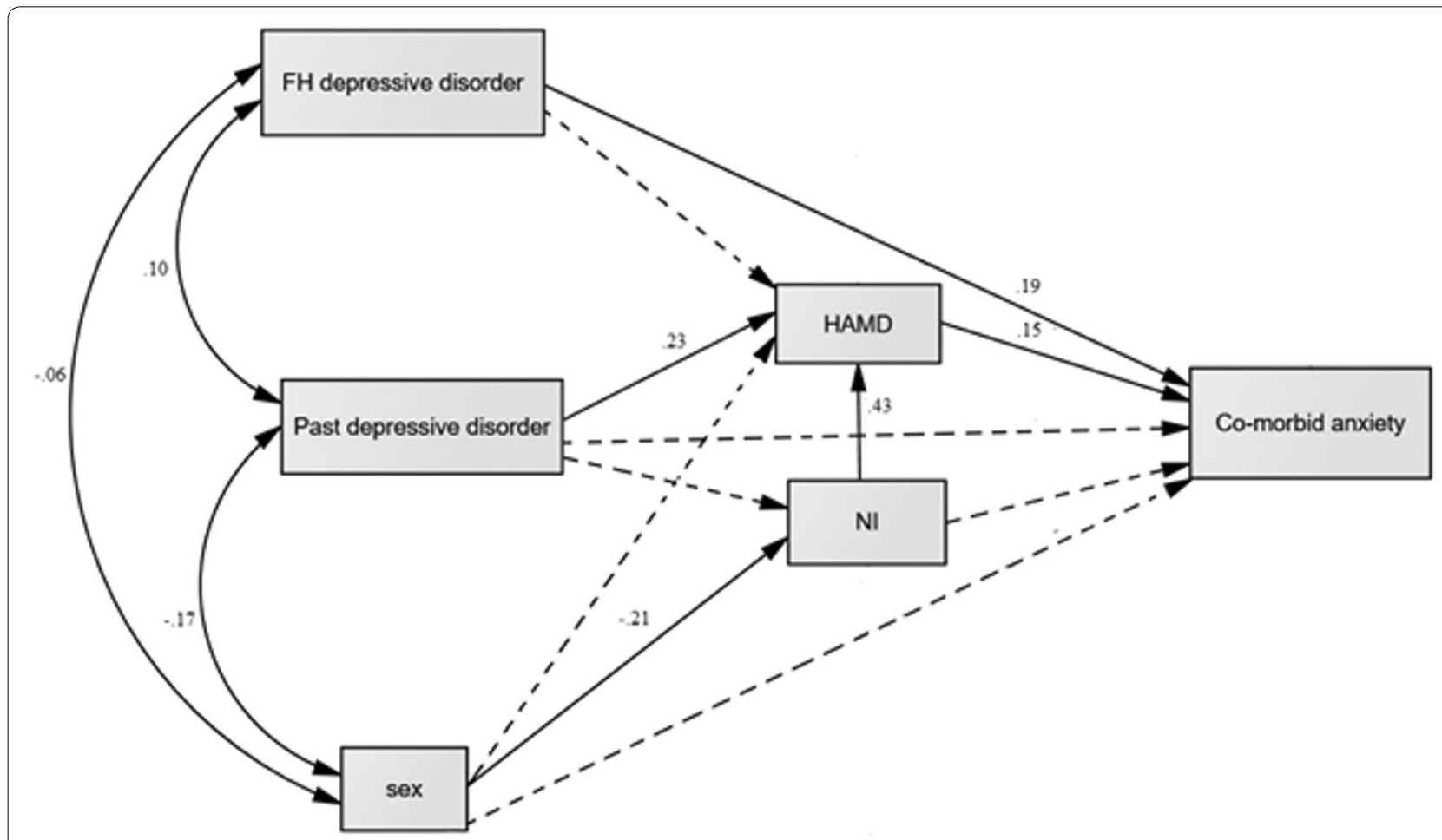

Fig. 2 The final path model illustrating direct and indirect effects and causal paths linking variables with comorbid anxiety and depressive disorders. N/neuroticism Inventory, HAMD Hamilton Depression Rating Scale, FHfamily history of depressive disorder. The lines with arrowheads show the direction of the path coefficients. Values on the lines = significant path coefficient or standardized coefficient. Dash lines denotes nonsignificant path coefficient or standardized coefficient and the actual values omitted

Table 3 The standardized coefficients and bias-corrected $95 \%$ confidence interval of effect of variables on mixed anxietydepressive disorder

\begin{tabular}{lllllll}
\hline Variables & Total effect & $\boldsymbol{p}$-value & Direct effect & $\boldsymbol{p}$-value & Indirect effect & $\boldsymbol{p}$-value \\
\hline Gender & $-0.020(-0.046,-0.007)$ & 0.002 & - & - & $-0.020(-0.046,-0.007)$ & 0.002 \\
Past history of depression & $0.053(0.025,0.097)$ & 0.001 & - & - & $0.053(0.025,-0.094)$ & 0.001 \\
Family history of depression & $0.192(0.021,0.354)$ & 0.060 & $0.192(0.021,0.354)$ & 0.060 & - & - \\
HAMD score & $0.215(0.107,0.316)$ & 0.001 & $0.215(0.107,0.316)$ & 0.001 & - & - \\
Neuroticism & $0.092(0.041,0.153)$ & 0.001 & - & - & $0.092(0.041,0.153)$ & 0.001 \\
\hline
\end{tabular}


the reversed role of depression severity affecting the emergence of comorbid anxiety disorders. Suspected explanations for this might be the same effect of neurobiologic change of the HPA axis or neurotransmitter, together with common psychosocial factors that contribute to both depression and anxiety.

Secondly, neuroticism mediates various variables, namely sex, depressive history and family history of depressive disorder. With neuroticism as a "trait" that persists throughout one's life, it would be inevitable that this personality trait plays many roles across different aspects of a person's psychological making. Being female has been found to be associated with higher level of neuroticism, both in younger and older age groups [23], which could potentially explain the higher prevalence of comorbid anxiety disorder among older women compared with men. How neuroticism is associated with anxiety has been studied to an extent, among Western and Asian populations confirming its role in this contribution. Evidence suggests that neuroticism predisposes to both anxiety and depression. One interesting theory that endeavored to explain this association was how genetic factors overlapped between anxiety and neuroticism, resulting from a twin study applying the structural equation model [13]. With this theory in mind, it would be likely that genetics might also play a role in the mediation effect of family history of depressive disorder. Similarly, with neuroticism likely to persist through one's life span, and evidence shown that it is a strong risk factor for depression [24], neuroticism contributes to history of depressive disorder playing a role in the relationship with comorbid anxiety disorder.

Lastly, family history of depressive disorder had a direct effect on the comorbidity, possibly due to genetic susceptibility of both psychiatric disorders recently believed to be on the same spectrum. Molecular genetic studies have identified specific genetic loci that may have influenced this susceptibility, albeit in their preliminary stages [13].

This study is among the few and most extensive studies on the comorbidity of anxiety disorders in LLD among Asians. The strength of this study includes the fact that we used data from a respectable study conducted in tertiary care centers, including as many bio-psycho-social factors as were identified in related literature. Moreover, we created a path model to investigate further relationships among variables and tested our hypothesis regarding the mediation model. The main limitations of this study revolved around the cross-sectional design that limited a claim of causal relationship, and longitudinal study that should be conducted next. In addition, some of the data that could potentially have been included to explain our hypothesis were lacking, for example, family history of anxiety disorder. Further studies with more extensive data would prove beneficial.

\section{Conclusion}

Neuroticism is an important mediator of comorbid anxiety disorder in LLD with effects on sex, family history and history of depressive disorder, providing supportive evidence for a theory emphasizing the complex relationships among depression, anxiety and neuroticism. This finding shows that neuroticism, strongly linked to anxiety, fear, worry and frustration, core components of all anxiety disorders, as well as depressed mood, explains why some variables are related to the increased rate of comorbidity. Moreover, depression severity is a mediator for neuroticism, as reflected by HAMD-7 scores. A longitudinal study should be conducted to further ascertain the effect of these variables that could be used to create appropriate interventions to lower the risk of developing comorbid anxiety disorders.

\section{Abbreviations \\ M.I.N.I.: Mini-International Neuropsychiatric Interview; NI: Neuroticism inventory; HAMD-7: The 7-item Hamilton Rating Scale for Depression; SEM: Structural equation modeling; CFI: Comparative fit index; TLI: Tucker-Lewis index; RMSEA: Root mean square error of approximation; CAIC: Consistent Akaike information criterion (CAIC); BIC: Bayesian information criteria; BCa: The bias-corrected and accelerated.}

\section{Acknowledgements}

The authors acknowledge the National Research Council of Thailand for funding support, endorsed by the Medical Research Network of the Consortium of the Thai Medical schools (MedResNet).

\section{Authors' contributions}

CS, NW, TW, PL, SJ, UT, SL, and SA designed the conceptualization. PK made data cleansing. CS, NW, and TW analyzed and interpreted the data and were major contributors in writing the manuscript. All authors read and approved the final manuscript.

\section{Funding}

The primary study (DAS program) was funded by the National Research Council of Thailand.

\section{Availability of data and materials}

The datasets used and/or analyzed during the current study are available from the corresponding author upon reasonable request as data sharing is subject to Ethics Office approval.

\section{Ethics approval and consent to participate}

This project was approved by Central Research Ethics Committee of Thailand, the ethics committee of Prasat Neurological Institute, the ethics committee of Songkhla Rajanagarindra Psychiatric Hospital, and the independent research committee of Faculty of Medicine, Chiang Mai University. All participants were informed about the study and provided informed consent.

Consent for publication

Not applicable.

Competing interests

The authors declare that they have no competing interests. 


\section{Author details}

${ }^{1}$ Geriatric Psychiatry Unit, Department of Psychiatry, Faculty of Medicine, Chiang Mai University, 110 Intawaroros Rd., Chiang Mai 50200, Thailand.

${ }^{2}$ Department of Family Medicine, Faculty of Medicine, Chiang Mai University, Chiang Mai, Thailand. ${ }^{3}$ Songkhla Rajanagarindra Psychiatric Hospital, Songkla, Thailand. ${ }^{4}$ Prasat Neurological Institute, Bangkok, Thailand. ${ }^{5}$ Department of Psychiatry, Faculty of Medicine, Khon Kaen University, Khon Kaen, Thailand.

Received: 25 May 2019 Accepted: 25 October 2020

Published online: 17 November 2020

\section{References}

1. Lenze EJ, Mulsant BH, Shear MK, Schulberg HC, Dew MA, Begley AE, et al. Comorbid anxiety disorders in depressed elderly patients. Am J Psychiatry. 2000;157(5):722-8. https://doi.org/10.1176/appi.ajp.157.5.722.

2. van der Veen DC, van Zelst WH, Schoevers RA, Comijs HC, Voshaar RC. Comorbid anxiety disorders in late-life depression: results of a cohort study. Int Psychogeriatr. 2015;27(7):1157-65. https://doi.org/10.1017/ S1041610214002312.

3. Mulsant BH, Reynolds CF, Shear MK, Sweet RA, Miller M. Comorbid anxiety disorders in late-life depression. Anxiety. 1996;2(5):242-7. https://doi. org/10.1002/(SICI)1522-7154(1996)2:5\%3c242::AID-ANXI6\%3e3.0.CO;2-O.

4. Prina AM. Co-occurrence of anxiety and depression amongst older adults in low-and middle-income countries: findings from the 10/66 study. Psychol Med. 2011;41:2045-56. https://doi.org/10.1017/S00332917110004 44.

5. Zhang X, Norton J, Carrière I, Ritchie K, Chaudieu I, Ancelin ML. Generalized anxiety in community-dwelling elderly: prevalence and clinical characteristics. J Affect Disord. 2015;172:24-9. https://doi.org/10.1016/j. jad.2014.09.036.

6. Almeida OP, Draper B, Pirkis J, Snowdon J, Lautenschlager NT, Byrne G, et al. Anxiety, depression, and comorbid anxiety and depression: risk factors and outcome over two years. Int Psychogeriatr. 2012;24(10):1622-32. https://doi.org/10.1017/S104161021200107X.

7. Hofmeijer-Sevink MK, Batelaan NM, van Megen HJ, Penninx BW, Cath DC, van den Hout MA, van Balkom AJ. Clinical relevance of comorbidity in anxiety disorders: a report from the Netherlands Study of Depression and Anxiety (NESDA). J Affect Disord. 2012;137(1-3):106-12. https://doi. org/10.1016/j.jad.2011.12.008.

8. Meier SM, Petersen L, Mattheisen M, Mors O, Mortensen PB, Laursen TM. Secondary depression in severe anxiety disorders: a population-based cohort study in Denmark. Lancet Psychiatry. 2015;2(6):515-23. https://doi org/10.1016/S2215-0366(15)00092-9.

9. Khan AA, Jacobson KC, Gardner CO, Prescott CA, Kendler KS. Personality and comorbidity of common psychiatric disorders. Br J Psychiatry. 2005;186:190-6. https://doi.org/10.1192/bjp.186.3.190.

10. Jeste ND, Hays JC, Steffens DC. Clinical correlates of anxious depression among elderly patients with depression. J Affect Disord. 2006;90(1):3741. https://doi.org/10.1176/appi.ajp.161.9.1581.

11. Kessler RC, MCGonagle KA, Zhao S, Nelson CB, Hughes M, Eshleman S, et al. Lifetime and 12-month prevalence of DSM-III-R psychiatric disorders in the United States. Results from the National Comorbidity Survey. Arch Gen Psychiatry. 1994;51(1):8-19. https://doi.org/10.1001/archp syc.1994.03950010008002.
12. Kendler KS. Major depression and generalised anxiety disorder. Same genes, (partly)different environments—revisited. Br J Psychiatry. 1996 https://doi.org/10.1192/S0007125000298437.

13. Hettema JM, Prescott CA, Kendler KS. Genetic and environmental sources of covariation between generalized anxiety disorder and neuroticism. Am J Psychiatry. 2004;161(9):1581-7. https://doi.org/10.1176/appi. ajp.161.9.1581.

14. Steffens DC, Wu R, Grady JJ, Manning KJ. Presence of neuroticism and antidepressant remission rates in late-life depression: results from the Neurobiology of Late-Life Depression (NBOLD) study. Int Psychogeriatr. 2018;30:1069-74

15. Canuto A, Giannakopoulos P, Meiler-Mititelu C, Delaloye C, Herrmann FR, Weber K. Personality traits influence clinical outcome in day hospital-treated elderly depressed patients. Am J Geriatr Psychiatry. 2009; 17:335-43.

16. Hayes AF. Introduction to mediation, moderation, and conditional process analysis: a regression-based approach. New York, NY, US: Guilford Press; 2013.

17. Wongpakaran N, Wongpakaran T, Lerttrakarnnon $P$, Jiraniramai S, Sirirak T, Assanangkornchai S, Taemeeyapradit U, Tantirangsee N, Lertkachatarn S, Arunpongpaisal S, Kuntawong P. Prevalence, clinical and psychosocial variables of depression, anxiety and suicidality in geriatric tertiary care settings. Asian J Psychiatr. 2019;41:38-44.

18. Kittirattanapaiboon P, Khamwongpin M. The validity of the Mini-International Neuropsychiatric Interview (M.I.N.I):-Thai version (13-1). Chiang Mai: I Am Organizer and Advertizing, Ltd.; 2004

19. McIntyre RS, Konarski JZ, Mancini DA, Fulton KA, Parikh SV, Grigoriadis S, et al. Measuring the severity of depression and remission in primary care: validation of the HAMD-7 scale. Can Med Assoc J. 2005;173(11):1327-34. https://doi.org/10.1503/cmaj.050786.

20. Wongpakaran $\mathrm{N}$, Wongpakaran $\mathrm{T}$. The effect of neuroticism on academic performance and quality of life in third-year medical students, Chiang May University. Chiang Mai, Thailand: Department of Medicine, Chiang Mai University; 2012. https://doi.org/10.1017/CBO9781139176200.

21. McCrae RR, Costa PT. The five-factor theory of personality: theoretical perspective. In: Pervin LA, John OP, editors. Handbook of personality: theory and research. 2nd ed. New York: Guilford; 1999.

22. Baron RM, Kenny DA. The moderator-mediator variable distinction in social psychological research: conceptual, strategic, and statistical considerations. J Pers Soc Psychol. 1986;51(6):1173-82. https://doi. org/10.1037//0022-3514.51.6.1173.

23. Chapman BP, Duberstein PR, Sörensen S, Lyness JM. Gender differences in five factor model personality traits in an elderly cohort: extension of robust and surprising findings to an older generation. Pers Individ Differ. 2007;43(6):1594-603. https://doi.org/10.1016/j.paid.2007.04.028.

24. Alnaes $\mathrm{R}$, Torgersen S. Personality and personality disorders predict development and relapses of major depression. Acta Psychiatr Scand. 1997;95(4):336-42. https://doi.org/10.1111/j.1600-0447.1997.tb09641.x.

\section{Publisher's Note}

Springer Nature remains neutral with regard to jurisdictional claims in published maps and institutional affiliations.

Ready to submit your research? Choose BMC and benefit from

- fast, convenient online submission

- thorough peer review by experienced researchers in your field

- rapid publication on acceptance

- support for research data, including large and complex data types

- gold Open Access which fosters wider collaboration and increased citations

- maximum visibility for your research: over 100M website views per year

At BMC, research is always in progress.

Learn more biomedcentral.com/submissions 